\title{
Modeling of the Human Orbit from MR Images
}

\author{
Zirui $\mathrm{Li}^{*}{ }^{*}$, Chee-Kong Chui ${ }^{1}$, Yiyu Cai ${ }^{2}$, Shantha Amrith ${ }^{3}$, Poh-Sun Goh ${ }^{4}$, \\ James H. Anderson ${ }^{5}$, Jeremy Teo ${ }^{1}$, Cherine Liu ${ }^{6}$, Irma Kusuma ${ }^{6}$, \\ Yee-Shin Siow ${ }^{6}$, Wieslaw L. Nowinski ${ }^{1}$ \\ ${ }^{1}$ Biomedical Imaging Lab, Singapore \\ ${ }^{2}$ School of MPE, Nanyang Technological University, Singapore \\ ${ }^{3}$ The Eye Institute, National University Hospital, Singapore \\ ${ }^{4}$ Dept. Diagnostic Imaging, National University Hospital, Singapore \\ ${ }^{5}$ Johns Hopkins University School of Medicine, Baltimore, USA \\ ${ }^{6}$ School of Computer Engineering, Nanyang Technological University, Singapore \\ lizirui@lit.a-star.edu.sg
}

\begin{abstract}
We previously described a parametric eyeball modeling system for real-time simulation of eye surgery (MICCAI 01). However, in the simulation of ophthalmologic surgery, the model of the eyeball alone is not sufficient. The orbital structures are as important as the eyeball. In this paper, we describe the approach to model the orbital structures from patient specific MRI data set and integrate the orbital model with the parametric eyeball model. The orbital tissues including the eyeball, muscles, and orbital fat are segmented from MRI data. An interactive image-based geometrical modeling tool is developed to generate a finite element model of the orbit. Preliminary results include biomechanical models of three human subjects, one of which is a young patient with a benign tumor in the right orbit. The biomedical model can provide quantitative information that is important in diagnosis. It can also be used to accurately analyze the result of intervention, which is an important component of the simulator for training and treatment planning. Our analysis includes a deformation study on an eyeball subjected to simulated tumor growth using the finite element method.
\end{abstract}

\section{Introduction}

Eye surgery, which is a specialty of microsurgery, involves very delicate tissues. The surgery is often performed relying on the magnified eye structure. Performing such a procedure requires a thorough knowledge of the clinical diagnosis and a very careful and accurate execution. Complications of ophthalmologic diseases, however, often pose additional difficulties for surgeons when making critical decisions. Very intensive training is required for ophthalmologic professionals in order to provide high quality standard surgical services.

Current imaging techniques are able to generate 3D data of the patient. In imaging the orbit, MRI provides considerable information of different soft tissues. The surgeons must investigate these data, slice by slice, and try to identity the pathology. However, without a patient specific model of the eye tissues, even the most experienced experts face difficulty in planning interventions.

In order to help the ophthalmologist to examine the patient specific data, and to help the student to get a better understanding of the related anatomy and surgery, we have 
developed a system to construct biomechanical model of the human orbit. Such model contains the eyeball, the muscles responsible for eye movement, the optic nerve, and the fat which fills the spaces in between. The model is aimed to help the ophthalmologist in different ways:

Quantification. The patient-specific model is able to provide information on the sizes, thickness, or volumes of different tissues. Such quantitative information is very important to the ophthalmologists in certain pathological conditions such as thyroid eye disease and in the cases of orbital trauma.

Diagnosis. The model can help the surgeons to better identify the abnormalities. If a certain disease is suspected to be developing, such a process could be detected and monitored through scanning and modeling of the patient data as a function of time. For example, creating 3D views of tumors in the orbit can enable the surgeon to understand the proximity and the exact relationship of the lesion to vital structures so that a more accurate diagnosis could be achieved.

Analysis. Along with proper material properties, the patient-specific model could be analyzed with FEM or other tools. The behavior of the eye tissues when interacted with surgical instruments can be accurately simulated. A more realistic simulation of intervention can be achieved.

Training. With the predefined models and result of the surgical procedure, the model can play an important role in training. If a virtual reality system is set up properly with some friendly graphic and haptic user interfaces, the trainee can obtain first hand experience and the training cost and its duration can be decreased. Moreover, orbital surgery is very highly specialized and training in such models can equip surgeons with the necessary skills to do a safe and efficient surgery.

Pretreatment planning. Such a model can help the surgeon to work out a better pretreatment plan. As in the case of brain, surgery in the orbit has very small margin for error. Practice with a virtual model can certainly help to reduce the risk of complications. Small incision surgery or stereotactic surgery in the orbit can be made possible in the future (such techniques are not available for orbital surgery at the moment) if a 3D image of the orbit and the pathological lesion is constructed.

At present time, several medical simulators have been reported [1-7]. For example, Hanna et al described their simulation work of accurate keratotomy for astigmatism [2]. Sagar et al built an eye surgical simulator within a virtual reality environment [3]. In this paper we discuss the patient specific modeling of the human orbit and how this model can be used to expand our virtual reality eye surgical simulation system [1].

\section{$2 \quad$ Patient Specific Orbital Modeling}

Current MRI scanners are able to scan the patient data at a resolution of $0.5 \mathrm{~mm}$ in three dimensions. However, the acquiring of the higher resolution data needs a longer time. If the scanning objects move or undergo deformation, distortion will be introduced. Because our eye is moving almost all the time, the scanning process should be finished as fast as possible. Therefore, the MRI data for the eye usually comes with a moderate resolution of, for example, $1.0 \mathrm{~mm}$. This resolution is good enough to work out the geometric model for the eyeball, muscles, nerves, and orbital bones. However, to define an image-based model for the internal structure of the eyeball, such a resolution is insufficient. In our approach, we construct an image- 
based model for the muscles, nerve, fat, and eyeball as a whole. For the structures contained in the eyeball, we employ a parametric model. This allows us to build up a patient specific model suitable for simulation and analysis. Figure 1 illustrates our approach.

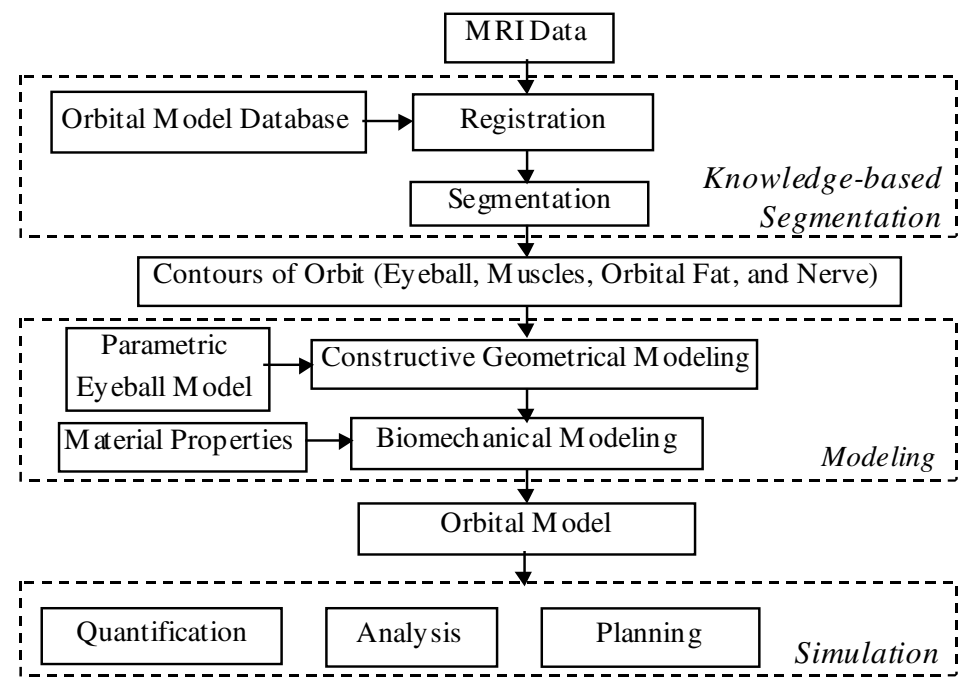

Fig. 1. Orbital modeling for ophthalmologic simulation

\subsection{Knowledge-Based Segmentation}

Our approach employs a database of orbital models in extraction of the orbital tissues from MR images. Currently, we have three MRI volumetric scans of human orbits including both left and right eyes. The first set is an infant with a diseased right eye. The remaining two sets are volunteer adults with no apparent pathologies in either eye. The voxel sizes are about $1.0 \times 1.0 \times 1.0 \mathrm{~mm}$ for the adults and $0.625 \times 0.625 \mathrm{x}$ $0.625 \mathrm{~mm}$ for the infant. Since the MRI data set does not contain sufficient information on the internal structures within the eyeball, in our segmentation work, we have to treat the eyeball as a single object in segmentation.

This virtual orbital model includes the eyeball, muscles, and the outer boundary of the orbital fat, which is similar to the shape of a cone. The critical feature points in the patient data such as the anterior and posterior end of the eyeball and the tip of the geometrical cone are used to develop a rough correspondence between the patient data and the virtual model. After that, the contour of the tissue from the virtual model is displayed on top of each slice of the patient data slices in axial, sagittal and coronal directions, respectively. The user will identify and confirm the exact position and shape of such tissues. Because a predefined model is used in segmentation and modeling, our method is knowledge-based.

To implement knowledge-based segmentation, a suitable software tool is developed. Using this software, registration and segmentation can be performed interactively. The user can adjust shapes of the virtual model to make it fit as much as possible to the patient data. 
Adjustment of the shape tissue is implemented through interactive region growing. If a region is identified as a particular structure and its boundary is not the same as the one defined by the virtual model, the user can adjust boundary of the virtual model manually. This can be done by changing the position of a control point and/or generating a new one through interactive region growing. Figure 2 (a) and (b) illustrates the contour of optic nerve before and after adjustment through region growing.

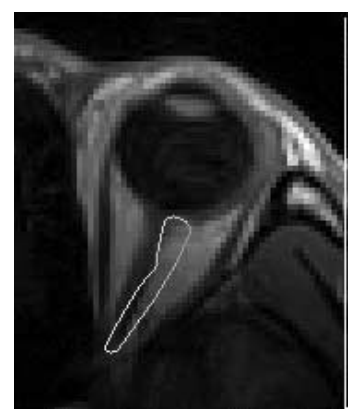

(a)

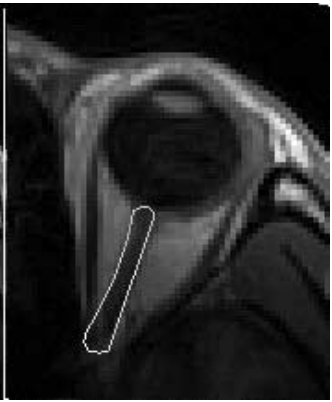

(b)

Fig. 2. (a) initial postion of optic nerve from the model database (b) after editing

\subsection{Modeling}

The main structures from segmentation process consist of the eyeball (EB), optic nerve (ON), superior rectus (SR), inferior rectus (IR), medial rectus (MR) and lateral rectus (LR). In addition to these structures, the outer boundary of the soft orbital tissues is also defined. We have considered these orbital tissues as orbital fat (OF). There might also be tumor in addition to the orbital fat. All the structures are segmented separately, and are regarded as independent objects. These objects are combined to obtain a single human orbital model.

At this stage, the eyeball is modeled as a unique, homogenous $3 \mathrm{D}$ structure. The reason is that the resolution of the patient specific MRI data is not high enough to segment the isolated eyeball tissues for modeling and analysis. In our previous work, we have modeled the eyeball parametrically[1].. Individual parts of the eyeball were parametrically represented and the variation of the geometry was possible. Figure 3 illustrates the polygonal eyeball representation. Through adjusting the parameters, the parametric eyeball is fitted with the segmented contours of the eyeball from the patient. A full geometrical model of the eyeball can be constructed.

The parametric eyeball, together with the other component models, contributes to our virtual orbital model. Figure 4 shows an example of the component models.

From these intermediate geometrical models, quantitative information such as dimension and volume of the various components could be determined. If further biomechanical analysis is required, the material properties are assigned to each component model that represents the respective anatomical structure. The resultant biomechanical model is used to update the orbital model database. 

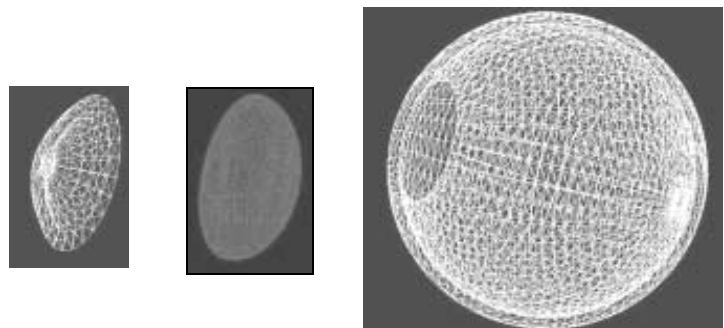

Fig. 3. Parametric representation of eyeball model

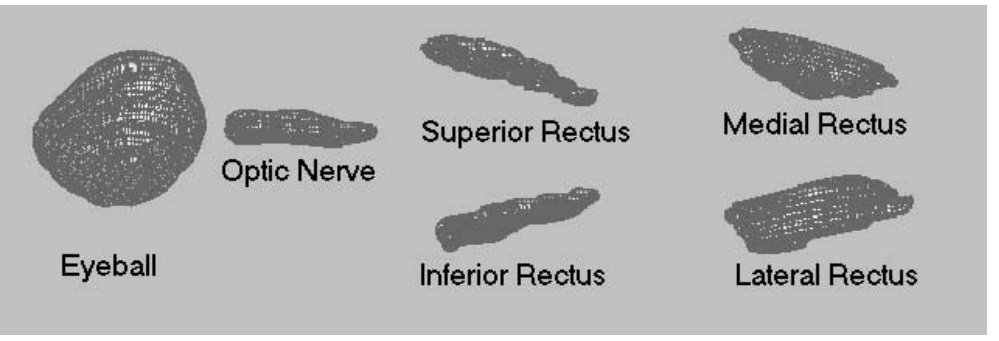

Fig. 4. Component models of various anatomic structures

\section{Finite Element Analysis}

\subsection{Biomechanical Properties}

Studies have shown that $90 \%$ of corneal thickness is formed by the stroma. We assumed that this controls the majority of the biomechanical behavior of the cornea, which consists mainly of water (78\%) and layered protein fibres (16\%). For such materials, the stress-strain relationship is nonlinear. We take the formula introduced by Uchio et al. [8]. Compared with other soft tissues in the orbit, the orbital bone could be defined as rigid. In our model, the functions of the orbital bones are implemented through the application of appropriate boundary conditions. As listed in Table 1, the physical properties of aqueous, vitreous, and fatty tissues are taken from Power et al [9] and Todd et al[10].

Table 1. Material Properties of different orbit tissues

\begin{tabular}{|l|c|c|c|}
\hline \multicolumn{1}{|c|}{ Structure } & $\begin{array}{c}\text { Young's Modulus } \\
\boldsymbol{E}(\boldsymbol{M P a})\end{array}$ & $\begin{array}{c}\text { Poisson ratio } \\
\boldsymbol{v}\end{array}$ & $\begin{array}{c}\text { Density } \\
\boldsymbol{\rho}\left(\mathbf{k g} / \mathbf{m}^{\mathbf{3}}\right)\end{array}$ \\
\hline Cornea & Nonlinear & NA & 1400 \\
\hline Sclera & Nonlinear & NA & 1400 \\
\hline Lens & Rigid & NA & 315 \\
\hline Ciliary's body & 20 & 0.40 & 1600 \\
\hline Aqueous & 0.037 & 0.49 & 999 \\
\hline Vitreous & 0.042 & 0.49 & 999 \\
\hline Muscles & 20 & 0.40 & 1600 \\
\hline Fatty tissue & 0.047 & 0.49 & 999 \\
\hline Orbital bone & Rigid & NA & NA \\
\hline
\end{tabular}




\subsection{Governing Equation of Deformation of Biostructures}

In our previous work [1], we considered small incremental deformation involved in the interaction of point surgical device and the virtual eyeball model. It could calculate the deformation of the tissue and the force feedback from the scalpel before cutting happens. Linear analysis is performed.

In the current model, we use non-linear analysis to determine the deformation over the outer surface of the eyeball subjected to an incremented contact force from a growing tumor in the orbit. Because the stress-strain relationships of some of the materials in the human tissues are nonlinear, which means that the stiffness is a function of the deformation, the problem can be expressed as

$$
\mathrm{K}(d) d=Q
$$

where,

$$
\begin{array}{r}
\mathrm{K}=!_{i=1}^{N}{ }_{0}^{V_{i}} B^{T} D(d) B d v_{i} \\
d=\left[d_{1 x} d_{1 y} d_{1 z}, d_{2 x} d_{2 y} d_{2 z}, \quad, d_{N x} d_{N y} d_{N z}\right]^{T} \\
Q=\left[q_{1 x} q_{1 y} q_{1 z}, q_{2 x} q_{2 y} q_{2 z}, \quad, q_{N x} q_{N y} q_{N z}\right]^{T}
\end{array}
$$

In the above equations, $d$ is the displacement vector, $\mathrm{K}$ is the global stiffness matrix and $\mathrm{Q}$ is the force vector applied to the orbit structure.

In order to solve these equations, $K$ and $d$ have to be built and solved step by step repeatedly. In our system, we adopted a load increment algorithm, in which the load is applied by several steps. If we define,

$$
\Delta q_{m}=Q_{m+1}-Q_{m}
$$

the iterative displacement change can be obtained using,

$$
d_{m+1}-d_{m}=\left(K_{T}\right)_{m}^{-1} \Delta q_{m}
$$

\section{$4 \quad$ Results and Discussions}

As previous work, Yu et al [11] has built a 3D orbital model from Visible Human Female data set and Scheimpflug images. They segmented the orbital tissue manually for the purpose of visualization and atlas construction. But it is not completed enough for the purpose of surgical simulation. Existing ophthalmic simulators, such as [1-7], define their ophthalmic models either geometrically and/or physically. These simulators simulate retinal coagulation, cataract surgery, and vitrectomy. They are mainly concentrated on the eyeball. There are, however, numerous procedures in the orbital region [12]. To our best knowledge, there is no orbital simulator using patientspecific data. Our work contributes towards building a suitable simulator for this purpose. The biomedical model we re-constructed from the patient MRI data set includes the geometrical structure and the definition of the physical properties of tissues. The 3D geometrical model serves the simulation system in the aspect of realistic visualization, which is one of the most critical components of the medical 
simulator. Quantitative information such as thickness and volume are also important to the ophthalmologist in his/her diagnosis and treatment planning. In addition, the physical-based model provides the displacement or deformation of the orbit tissues under interventions. The force feedback calculated from the model can be applied to the haptic devices to achieve realistic force feedback.

Table 2. Examples of orbital models and their volume in $\mathrm{mm}^{3}$

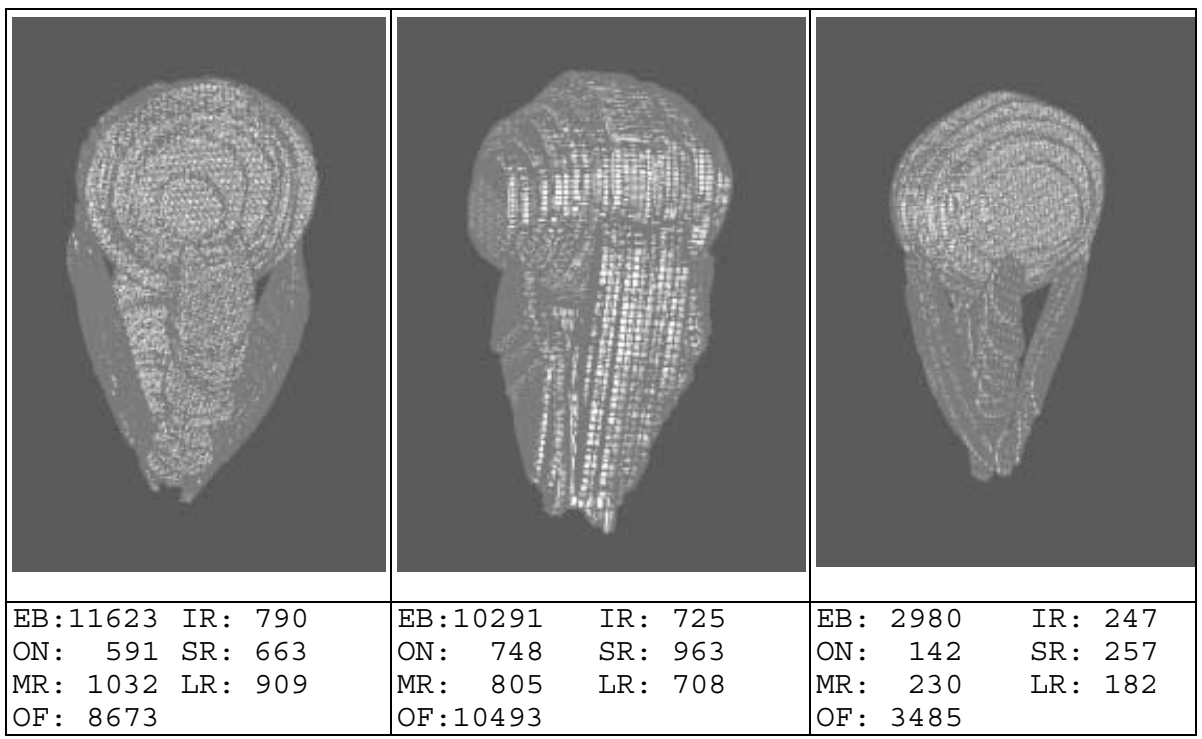

Table 2 is a snap shot of the orbital models and the volume of the different tissues in the three orbital models. In this table, the volume of orbital fat includes the soft tissues other than segmented and defined, such as the eyelid.

We have extended our analysis to the study of the deformation and stress distribution on pathological eyeball using our orbital model. Compared with other work based on the surface model, our 3D nonlinear model can yield better results because more accurate physical models are employed. Figure 5 shows the deformation of a patient's eyeball with a growing tumor at the orbit. Such results can help the clinicians in evaluating the pressure inside the orbit and take proper measures for diagnosis and treatment.

Essentially, the model represents the orbit structures in a static state when the eyes are closed and relaxed. This is the zero-stress state when the deformation and force feedback are calculated. However, the position of eyeball and the shape of muscles are changing all the time. A more flexible model is under development.

At the current stage, our FEM analysis does not include cutting and remeshing components. However, such functionalities are essential to ophthalmic surgery simulation. In addition, the current model is not completed yet. Many important structures such as blood vessels, nerves, and different orbital bones are not included yet. In the simulation point of view, quantitative validation has to be carried out on the speed of the system, accuracy of the model, etc. 


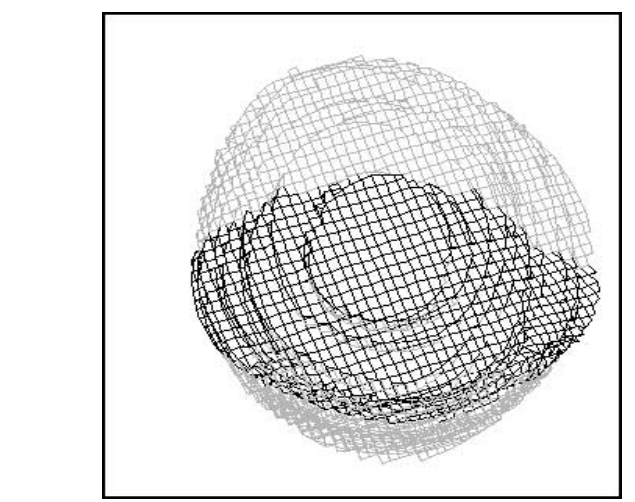

Fig. 5. Deformation of the eyeball under tumor pressure

\section{Conclusions}

We developed a method for the patient-specific modeling of the orbit. Such a method makes use of the available anatomic model to extract and build the patient-specific model. Confirmation of patient structure is done using interactive image processing, mainly $3 \mathrm{D}$ region growing. Using the published material properties, an analyzable FEM model is generated. A customized FEM algorithm is developed to analyze the behavior of the patient specific model. We demonstrated that our model could be used to study deformation in tumorous human orbit.

The orbital model can provide the ophthalmologist with: (1) quantitative information that is important in diagnosis; (2) a better way to identify the abnormalities; (3) a more accurate results in analyzing intervention and a more realistic simulation; (4) cost efficient training; and (5) better pretreatment planning through practicing with a virtual patient orbital model.

As future work, we are developing techniques to provide a really useful simulator. In addition to the functions such as cutting and remeshing, we are also work on the speeding up of analysis processes. Comparative evaluation between simulation results and real cases is also under way in order to evaluate the efficacy of the simulation system.

\section{Acknowledgements}

Support of this research development by the Agency for Science, Technology and Research, Singapore is gratefully acknowledged.

\section{References}

1. Cai Y, Chui CK, Wang Y, Wang Z, Anderson JH, Parametric eyeball model for interactive simulation of ophthalmologic surgery, MICCAI 2001, pp. 465-472

2. Hanna KD, Jouve FE, Waring GO, and Ciarlet PG, Computer simulation of accurate keratotomy for astigmatism, Refractive \& Corneal Surgery, Vol. 8, 1989, pp. 152-163. 
3. Schill MA, Wagner C, Hennen M, Bender HJ, Maenner R, EyeSi - a simulator for intraoccular surgery. MICCAI'99, pp. 1166-1174.

4. Sagar, M. A., Bullivant, D., Mallinson, G. D., Hunter, P. J., and Hunter, I. W., A virtual environment and model of the eye for surgical simulation, Proceedings of SIGGRAPH Annual Computer Graphics Conference 1994, Florida, pp. 205-211.

5. Parshall R. F., Computer-aided geometric modeling of the human eye and orbit. $J$. Biomedical Computing 1991, 18(2), pp. 32-39.

6. Li Z, Chui CK, Anderson JH, Chen X, Ma X, Hua W, Peng Q, Cai Y, Wang Y, Nowinski WL, Computer environment for interventional neuroradiology procedures, Simulation and Gaming, 2001, 32(3), pp. 405-420.

7. Peifer J. Virtual environment for eye surgery simulation. Medicine Meets Virtual Reality II: Interactive Technology and Healthcare, San Diego, 1994:166-173.

8. Uchio E, Ohno S, Kudoh J, Aoki K, Kisielewicz LT: Simulation model of an eyeball based on finite element analysis on a supercomputer. British Journal of Ophthalmology, Vol. 83, pp.1106-1111, 1999.

9. Power E. D., Stitzel J. D., West R. L., Herring I. P., and Duma S. M., A nonlinear finite element model of the human eye for large deformation loading, Proceedings of 25th Annual Meeting of Biomechanics, San Diego, August 2001, pp44-45.

10. Todd BA, Thacker JG, Three-dimensional computer model of the human buttocks in vivo, J rehabil. Res. Dev. 1994 31(2), pp.111-119.

11. Yu CP, Jagannathan L, Srinivasan R, Nowinski WL. Development of an eye model from multimodal data. Proceedings SPIE Medical Imaging 1998: Image Display. Vol. 3335, San Diego, California, Feb 1998, pp. 93-99.

12. Rootman J, Steward B., Goldberg RA. Orbital Surgery. A Conceptual Approach. Lippincott-Raven, Philadelphia, 1995. 